\title{
La maison nouméenne de Victor Segalen
}

\section{Un lieu de création littéraire?}

\author{
Eddy Banaré et Louis Lagarde \\ TROCA (Trajectoires Océaniennes), Université de la \\ Nouvelle-Calédonie
}

Victor Segalen (1878-1919), puissamment marqué durant son voyage en Océanie (1903-1904) par le monde polynésien, publie à son retour en Europe une de ses œuvres majeures: Les Immémoriaux (1907). La correspondance de Victor Segalen, écrite de Nouméa, Nouvelle-Calédonie, nous permet d'affirmer que le manuscrit de cet ouvrage, ainsi que les bases d'autres textes, dont le dossier Mythes polynésiens (non publié) et son Essai sur l'exotisme (publié après sa mort) ont été travaillés lors de l'escale de deux mois qu'il y fit en 1904. Ce séjour nouméen fut l'occasion pour lui de renouer avec un ami de jeunesse, le calédonien Lucien Nielly, fonctionnaire colonial rencontré en Bretagne chez les jésuites. C'est en logeant dans la maison de fonction de son hôte et en profitant d'un climat calédonien plutôt maussade cette année-là, que Segalen trouva le temps de modeler ses manuscrits. Cette petite maison est connue d'après des photographies anciennes et se situait sur les hauteurs du Quartier Latin. Si elle n'existe plus aujourd'hui, la recherche de sa localisation exacte est l'objet de cet article. Ainsi, en partant des faits historiques et biographiques connus, nous pouvons mieux définir le lieu et les conditions de séjour calédonien de Segalen, et partant, démontrer l'importance de cet intermède calédonien, véritable creuset créatif ayant permis de lier le monde polynésien traditionnel et un regard métropolitain d'exception. Ceci nous permet également de renouveler la question des lieux de création littéraire en Océanie. Si la maison de James Norman Hall à Arue, Tahiti, et celle de Robert Louis Stevenson à Vailima, Samoa, sont aujourd'hui des sites historiques et patrimoniaux importants, qu'en est-il des lieux de passage ou de séjour, en particulier dans une Océanie souvent découverte à la faveur d'escales, nécessairement temporaires?

Mots clefs: Segalen, Les Immémoriaux, creation, Pacifique, maison, Nouméa, archéologie, colonization

Victor Segalen (1878-1919), deeply impressed by Polynesian culture during his voyage to Oceania (1903-1904), published one of his major works, Les Immémoriaux (1907), upon his return to Europe. Segalen's letters written from Nouméa, New Caledonia, testify that the manuscript of this work, as well as the bases of other texts, including the unpublished Mythes polynésiens and the posthumous Essai sur l'exotisme, were penned during his two-month stopover there in 1904. That stay in Nouméa was an opportunity for him to reunite with an old friend, New Caledonia-born Lucien Nielly, a colonial administrator he had met during his time in a Jesuit high school in Brittany. Segalen found the time to shape his manuscripts while staying in the Nielly family home and taking 
advantage of the rather ghastly weather in Nouméa that year. This small house is known from old photographs and was located on the heights of a Noumean suburb known as the Quartier Latin. While it no longer exists, the search for its exact location is the subject of this article. Starting from known historical and biographical facts, we can reveal the location of Segalen's accommodation and the conditions of his stay in Nouméa, and thus demonstrate the importance of this New Caledonian sojourn, a true crucible of creativity which allowed the fusing of the traditional Polynesian world and an exceptional French mindset. This will also allow us to shed new light on the question of places of literary creation in Oceania. If James Norman Hall's house in Arue, Tahiti, and that of Robert Louis Stevenson in Vailima, Samoa, are important heritage and historic sites today, how should we consider visited places or temporary dwellings, especially in Oceania, which was often encountered through necessary stopovers?

Keywords: Segalen, Les Immémoriaux, creation, Pacifique, maison, Nouméa, archéologie, colonization

La francosphère du Pacifique trouve ses origines dans les expéditions d'exploration de la seconde moitié du $\mathrm{I}{ }^{\text {ème }}$ siècle, puis dans l'œuvre des missionnaires francophones et les politiques d'expansion coloniale du siècle suivant. Elle constitue aujourd'hui, comme le montrent les contributions de ce volume, un espace puissamment interconnecté, marqué par les mobilités et les diasporas contemporaines. L'objet de cet article est de remonter aux premières années du $20^{\text {ème }}$ siècle, pour suivre les traces d'un jeune médecin de marine ayant traversé à plusieurs reprises, en 20 mois, l'essentiel du Pacifique francophone, à savoir les Îles-sous-le-Vent, les Tuamotu, les Marquises, Wallis (Uvea) et Nouméa: il s'agit de l'écrivain Victor Segalen (I878-1919). Son premier roman, Les Immémoriaux (I907), est aussi le seul publié de son vivant. ${ }^{1}$ Plus particulièrement, cet ouvrage constitue le cœur de sa production océanienne, aussi appelée Cycle Polynésien. Cuvre totalement atypique pour la période, Les Immémoriaux se situent à la confluence de la collecte ethnographique, du témoignage historique et du roman, tout en plaçant le lecteur face à un narrateur apparemment maori derrière lequel Segalen s'efface. Le lecteur métropolitain est ainsi mis au pied du mur, contemplant la déliquescence d'un monde qui s'enfuit à cause de l'évangélisation, corollaire de l'expansion coloniale.

L'étude de ce texte est aujourd'hui riche de multiples articles ${ }^{2}$ et a intéressé de nombreux chercheurs en littérature, en histoire et même en

I Victor Segalen (sous le pseudonyme de Max-Anély), Les Immémoriaux (Paris: Mercure de France, 1907).

2 Pour un ensemble récent, voir Colette Camelin, dir., "Exotisme et altérité: Segalen et la Polynésie ", Cahiers Victor Segalen, ${ }^{\circ} 2$ (Paris: Honoré Champion, 2015). 
anthropologie, la réédition dans la collection Terre Humaine de Plon en I956 ayant achevé de lui donner un statut quasi-documentaire. ${ }^{3}$ Toutefois, on notera que peu de travail a été fait localement, en Océanie, pour essayer de mieux comprendre les conditions de sa création. Nous nous intéresserons ici plus particulièrement aux trois séjours que Victor Segalen, médecin à bord de La Durance, effectue à Nouméa, Nouvelle-Calédonie, entre avril I 903 et octobre 1904. Au-delà de l'admiration pour l'auteur d'une œuvre exceptionnelle, l'intérêt pour le séjour calédonien de Segalen obéit au projet de décrire la vie qui a pu être la sienne à Nouméa, ville que sa correspondance nous permet d'envisager comme un lieu de création littéraire. La vie coloniale menée par Segalen à Nouméa - que le recoupement des sources littéraires, testimoniales, photographiques et archivistiques nous a permis de restituer - peut-elle servir de révélateur à la genèse des Immémoriaux?

Nous explorerons d'abord la genèse calédonienne de l'œuvre, en récapitulant les faits déjà connus, en explorant de manière exhaustive la correspondance publiée chez Fayard en $2004,{ }^{4}$ en détaillant les relations de Segalen à Nouméa, plus particulièrement la famille Nielly qui l'héberge. La localisation de l'habitation Nielly, lieu de création littéraire, sera ensuite présentée, avec l'importance archéologique et littéraire qui en découle. Enfin, la valorisation de ce lieu de création fugace, dont il ne subsiste aujourd'hui que peu de choses, est à proposer, même si Victor Segalen n'éprouvait que des sentiments globalement négatifs vis-à-vis du chef-lieu de la colonie. Cette petite ville, avec son cadre contraignant et son peu de distractions, a ainsi permis à ce jeune homme de mettre en forme une des œuvres littéraires les plus audacieuses publiées à ce jour sur l'Océanie.

Christian Doumet et Marie Dollé, dans la préface rédigée pour l'édition de $200 \mathrm{I}$ des Immémoriaux, décrivent une écriture placée sous le signe de la nostalgie - celle d'un pays maori perdu - et de l'absence - celle de Paul Gauguin, mort peu de temps avant l'arrivée de Victor Segalen aux Marquises. ${ }^{5}$ La chronologie établie ici suggère au moins deux séquences d'écriture: l'une, océanienne, mais postérieure au séjour tahitien, pendant laquelle Segalen aurait pris des notes et élaboré le plan du récit. Une seconde est située entre son retour en France en 1905 et la publication du texte au Mercure de France en 1907. En bref, on pouvait se trouver à spéculer sur une

3 Cette perception de l'œuvre est controversée, comme en témoigne l'opinion de Jean Guiart, Bêtisier océanien: ce qu'il faut savoir de l'Océanie et dictionnaire des erreurs courantes (Papeete/Nouméa: Le Rocher à la voile, 2013).

4 Victor Segalen, Correspondance, 3 volumes (Paris: Fayard, 2004).

5 Nous faisons référence à l'édition publiée par Le Livre de Poche dans la collection Les Classiques de Poche. 
écriture maritime, imaginant l'auteur, alors médecin de marine, travaillant à son manuscrit à bord de La Durance qui, après l'avoir conduit à Tahiti, le ramenait en France où il achèvera l'écriture du roman. Plus connue est, en revanche, la période allant de 1906 jusqu'à l'été 1907 pendant laquelle Segalen reprend le manuscrit, espérant le publier à temps pour le présenter aux jurés du Goncourt. ${ }^{6}$

Les conditions d'écriture océanienne des Immémoriaux sont donc demeurées assez floues jusqu'à la publication en 2004 de la correspondance de Segalen. ${ }^{7}$ Celui-ci est resté dans le Pacifique de janvier 1903 à septembre 1904. S'il est principalement stationné à Tahiti sur ces vingt mois en Océanie, il en passe trois à Nouméa, à l'occasion de plusieurs séjours. Le recoupement de plusieurs sources ${ }^{8}$ permet de préciser ces derniers: un premier passage du 23 avril I903 au I6 mai I903; un deuxième du 25 avril I904 au 2I juin I904; et un dernier du I4 septembre au 26 septembre I904. Ce temps passé en Nouvelle-Calédonie a plutôt été sous-estimé dans les travaux biographiques et le plus souvent réduit aux escales de La Durance. ${ }^{9}$ Il faut dire que le Journal des îles, qui avant la publication de la Correspondance, constituait le principal fil conducteur du séjour océanien, s'interrompt entre le $\mathrm{I} 4$ avril et le $\mathrm{I}^{\mathrm{er}}$ octobre $\mathrm{I904}$, occultant ainsi les deux derniers passages calédoniens. Les lettres de Victor Segalen permettent désormais de reconstituer, pendant le deuxième séjour à Nouméa, huit semaines intenses et disciplinées consacrées à l'élaboration des Immémoriaux. On peut donc avoir un éclairage sur le Cycle Polynésien et réinscrire pleinement la genèse du roman dans l'espace océanien.

Dès 1903 , c'est de Nouméa, dans une lettre à son ami Emile Mignard ${ }^{10}$ (qu'il appelle «mon frère »), datée du 24 avril (premier séjour), que Segalen brosse le récit qu'il entend produire sur Tahiti et qui s'appelle alors $L e$ Promeneur de nuit: $:^{11}$

6 Annie Joly-Segalen et Gabriel Germain, «Lettres inédites de Victor Segalen. L'entrée de Segalen au Mercure de France. Le Prix Goncourt 1907 ", Annales de Bretagne, 71.3 (1964), $429-43$.

7 La publication de la correspondance en trois volumes, chez Fayard en 2004, a permis l'accès à une documentation riche de plus de I,500 lettres, dont I,300 étaient à ce jour inédites.

8 Notamment Patrick O'Reilly, Tahitiens: répertoire bio-bibliographique de la Polynésie Française (Paris: Société des Océanistes, I975); Victor Segalen, Journal des îles, suivi de Vers les sinistrés, préface d'Annie Joly-Segalen (Papeete: Les Éditions du Pacifique, I978); ainsi que Segalen, Correspondance.

9 Voir à ce sujet l'introduction d'Annie Joly-Segalen au Journal des îles, pp. I3-I4.

Io Emile Mignard est un musicien, ami de jeunesse de Victor Segalen. C'est lui qui présentera au jeune Victor sa future épouse, Yvonne Hébert. Les jeunes hommes entretiennent une correspondance nourrie pendant environ Io ans, de I895 à 1905. Voir Segalen, Correspondance, Vol. I.

II Ce titre sera d'ailleurs utilisé dans les premières éditions pour l'un des chapitres des 
J'ai une certaine pudeur à parler de projets qui ne sont que projets. Pour toi elle disparaît. Et voici la " réalisation " qui me hante:

Titre: "Le Promeneur de Nuit»

Un «harepo ", prêtre de Taaroa, initié à la société des "Arioï ", sent vaguement le monde Polynésien s'en aller ... Vers I820-I830; au moment où la civilisation meurtrière s'infiltre dans ces terres-enfants ... Il lutte, oppose sa cosmogonie à la Bible, puis il devine le Vide: cette absence de Passé, cette absence de documents écrits ... cette jeunesse puérile de sa race. Il va tenter de lui donner une Bible, une Tradition. Alors, émigrant volontaire, il s'embarque à travers la Polynésie. Mais inconsciemment, il a pris la route que ses ancêtres, venus de Java, avaient déjà sanctifiée; il visite les Lieux Saints de la gent Maorie, guidé seulement par le mot "Sawaï », dont il connaît la valeur hiératique, et qui est son guide. Il visite Raïatea, la Sainte, où sur le tertre de "Sawaï », le dieu Oro fonda la secte orgiaque des Arioï. Il pérégrine à Sawaï, des Samoa ... Mais, toujours le Vide sur le Passé. Une tempête le rejette sur la plus extrême des îles Polynésiennes, l'île de Pâques, où, un moment, il croit tenir son but ... (quelques statues géantes et des traces d'inscriptions) mais il revient à Tahiti, découragé. Il se civilise lui-même, s'aveulit, s'enlise, végète ... je voudrais que ce soit le roman de la race elle-même; et mon Harepo (ou Promeneur de Nuit, car c'est à l'entour des temples, la nuit, qu'on récitait les versets sacrés) sera fait de matériaux vécus. $^{12}$

Parmi les quatorze lettres connues et envoyées de Nouméa, la plupart concernent l'escale de huit semaines de I904, qui donne à Victor Segalen l'occasion de se plonger dans ses notes: il a, en effet, détesté Nouméa et a trouvé dans le travail littéraire une échappatoire à ce séjour vécu comme une contrainte:

L'ami Nielly, toujours très dévoué, m’a forcé à accepter l'hospitalité chez lui. Une chambre, une table, des papiers sur la table, et des bouquins; avec ça, on ne moisit nulle part. J'avance à grands coups mon manuscrit, ponctuel comme un comptable. Dix pages par jour. C'est encore une façon comme une autre de vivre à Tahiti l'ensoleillée, malgré les torrents d'eau de la Calédonie. $^{13}$

On trouve également:

Je profite de cet interminable séjour en une ville insipide pour rédiger,

Immémoriaux. Ibid., p. 505.

I2 Ibid., pp. 504-05.

I3 Lettre à ses parents, le 25 avril I904. Victor Segalen, "Deux séjours de Victor Segalen, à Nouméa, voici cinquante ans. Lettres inédites ", Journal de la Société des Océanistes, Iо. Iо (1954), I85-91 (p. I89). 
rédiger à outrance toutes les notes vécues intensément à Tahiti. Je me suis donné (installé chez l'ami Nielly, complaisant, naïf et puéril) des heures de bureaux, d'autres d'escrime. Pas de femmes. Pas de café. Pas de cheval. Pas de bateau. Le pays m'indiffère. Je n'en vis pas, le traverse; bonheur de revenir en France avec mon manuscrit avancé, et la satisfaction (enfin) d'un travail mené jusqu'au bout. Le premier. ${ }^{14}$

De même:

Pluie-Vents depuis six mois! Vie de bureau persistante. Les feuillets raturés s'accumulent; j'espère rentrer avec les $2 / 3$ de composés. Puis l'excitation du milieu, enfin retrouvée, les longues causeries efficaces, efficientes reprises (avec toi, Max, Saint-Pol ...), mon dernier chapitre, et la révision, le polissage, marcheront vite. Je voudrais avoir remis les épreuves avant ma prochaine campagne, de façon à clore en même temps un gros morceau de vie intense, être paré à de nouveaux spectacles, resensibilisé pour de nouvelles races et de nouveaux soleils. ${ }^{15}$

Puis:

Nouméa s'afflige de tous les ennuis d'une ville d'Europe. Moins toujours la grande ville. J'ai trouvé un piano et des partitions. Je m'astreins à deux heures de travail littéraire par jour. Et surtout, ce qui vaut mieux encore, à mettre ma vie pratique en accord avec ma vie artistique. ${ }^{16}$

Enfin:

Nouméa a ceci de morne: que c'est une stupide ville. Ceci de vide que je suis orphelin de courriers depuis trois mois. Ceci de troublant j’y ai découvert une troublante jeune fille triste, aux pâles yeux pers, désabusée et frêle; Nous nous écrivons des choses désolées, et préparons notre adieu.

Ponctuellement, je "couvre» cinq pages en 24 heures. Je vais plus vite depuis l'entraînement sérieux du dernier mois. Dire que cet élément métier, chronométrique et mesquin, est nécessaire, pour que la rêverie se réalise, et que l'idée se revête de Forme. ${ }^{17}$

La ville de Nouméa n'apparaît dans la correspondance que sous un jour négatif: sale et provinciale, trop européanisée à son goût, marquée par le sceau du bagne, elle se substitue à la Calédonie toute entière, dont Segalen

I4 Lettre à Emile Mignard, le Io mai I904. Segalen, Correspondance, Vol. I, p. 578.

I5 Lettre à Emile Mignard, le $5_{5}$ mai I904. Segalen, Correspondance, Vol. I, p. 579.

I6 Lettre à ses parents, le 2 juin I904. Segalen, Correspondance, Vol. I, p. 58I.

I7 Lettre à Emile Mignard, le I5 juin I904. Segalen, Correspondance, Vol. I, pp. 583-84. 
goûte par ailleurs peu les habitants, les jugeant trop différents de ses chers Tahitiens. Seuls crédits à ses yeux: la clarté cristalline de l'air et la qualité de la lumière calédonienne, les jours où il fait beau bien entendu. ${ }^{18}$ Sans surprise, c'est donc dans ces conditions qui s'apparentent à un cantonnement forcé qu'il travaille pour la première fois durablement à son manuscrit. Au-delà du peu d'intérêt qu'il a pour la Calédonie, le rythme de travail qu'il s'impose à Nouméa, et que l'on perçoit clairement à travers sa correspondance, démontre l'importance de ce séjour en termes de création littéraire.

Cette genèse est permise par la présence à Nouméa de Lucien Nielly (I880-I959), ami de jeunesse de Segalen. ${ }^{19}$ C'est à la fin de son premier séjour calédonien, qu'il mentionne le fait d'avoir renoué avec Nielly. ${ }^{20}$ De Tahiti, quelques temps avant son second séjour calédonien, il conseille à sa famille d'adresser le courrier directement chez Nielly, ce qui indique qu'il y habite dès son arrivée. ${ }^{21}$ Sur son dernier séjour d'une semaine, en septembre 1904, nous n'avons pas de témoignages directs quant au lieu où il séjourne mais il mentionne Nielly à Emile Mignard, disant que ce dernier est toujours égal à lui-même, sympathique et dévoué. Calédonien, Lucien Nielly est le petit-fils d'Arthur Pelletier, premier maire de Nouméa (I874-I879), suite à la création par le colonel Alleyron, gouverneur provisoire, du premier corps municipal de la ville. ${ }^{22}$ Fait rare à cette époque, Nielly a fait une partie de son cursus scolaire en France, au collège de Jésuites Notre-Dame du Bon Secours de Brest, où il rencontre Victor Segalen. ${ }^{23}$ Revenu en Nouvelle-Calédonie en I900, Lucien Nielly entre dans l'administration comme commis de $3^{\text {ème }}$

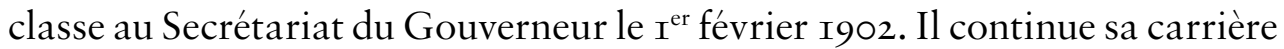
dans l'administration coloniale, étant tour à tour Commissaire-résident de

I8 Lettre à ses parents, le 24 avril I903: " Arrivée, dirai-je, lugubre: pays pelé par le soleil, avec l'anachronisme d'une pluie antédiluvienne. Une rade minable: on débarque. On patauge dans la boue, le poussier de charbon; comme aspect, une banlieue commerciale, grise, encombrée où l'on croise des mines patibulaires de forçats libérés, ou évadés, ou tout comme ». Segalen, "Deux séjours de Victor Segalen », p. I85.

I9 Cette amitié entre les deux hommes est connue par la correspondance de Victor Segalen mais aussi par son souvenir entretenu dans la mémoire familiale: Patrice, le fils de Lucien Nielly, artiste-peintre, avait témoigné de cette amitié dans la notice biographique qui lui est consacrée dans l'ouvrage de Bernard Villechalane et Jean-Jacques Syllebranque, Peintres de la Nouvelle-Calédonie, du Vanuatu et des Wallis (Nouméa: Les Éditions du Cagou, I98I), p. 73.

20 Lettre à ses parents du 28 avril I903, Segalen, "Deux séjours de Victor Segalen », p. I86.

2 I Segalen, Correspondance, Vol. I, p. 523.

22 Jean Lèques, dir., Des Maires, une ville: Nouméa I859-I986 (Nouméa: Édition Ville de Nouméa, 2004), p. 4.

23 O'Reilly, Tahitiens, p. 516. Le passage par cette institution semble correspondre à une tradition familiale chez les Nielly, notamment chez ceux qui se destinaient à une carrière d'officier de marine: communication personnelle avec Marie-Josée Nielly, Nouméa, septembre 2016. 
France aux Nouvelles-Hébrides par intérim (I9I8), puis nommé en 1932 chef de Bureau des Secrétariats Généraux de Guyane Française et de l'Inini, et enfin au Soudan Français (actuel Mali). C'est le I5 septembre I946 qu'il quitte Marseille sur le Ville d'Amiens pour revenir en Nouvelle-Calédonie, où il termine sa vie paisiblement, en $1959 .{ }^{24}$

La localisation de la maison de Lucien Nielly, dans laquelle Victor Segalen a séjourné, a été possible grâce aux témoignages familiaux, ${ }^{25}$ à la consultation des registres du Service de la Publicité Foncière de NouvelleCalédonie, ainsi qu'à la présence d'un album de photographies conservé au Service des Archives de la Nouvelle-Calédonie sous le ${ }^{\circ}{ }^{\circ} 48 F_{15}$. Cet album était donné comme étant celui de Charles Nielly, le père de Lucien Nielly. Toutefois, celui-ci étant décédé en I894, la paternité de l'album semble être celle d'Arthur Pelletier, grand-père maternel de Lucien Nielly et son tuteur légal jusqu'à sa majorité. Il contient plusieurs vues d'une même habitation en clins de bois, partiellement entourée d'une véranda et couverte d'un toit en tôle ondulée, datées de la période 1902-1907, dont une porte la légende "Mon Home à Nouméa en I902" (fig. I).

Ce cliché montre la façade Est de la maison, donnant sur la rue de Sébastopol. Il s'agit d'une maison classique de l'architecture coloniale calédonienne privée $e^{26}$ : ainsi on accède à la véranda par un petit escalier à volée simple au milieu de la façade, qui permet de joindre le jardin situé en contrebas et l'espace de vie, séparés par un vide sanitaire. Ce dernier permet la circulation de l'air sous la maison afin d'assécher ses éléments en bois et d'abaisser la température intérieure. On distingue de hautes portesfenêtres persiennées de l'autre côté de la véranda étroite, qui transforment celle-ci en voie de communication entre les pièces. Le jardin sur rue est étroit et densément planté, afin de se prémunir des regards extérieurs, avec un manguier (Mangifera indica) à gauche et un pêcher rustique (Prunus persica) à droite de la façade.

Un second cliché de l'album (fig. 2) montre l'arrière de la maison, au jour d'une réunion de famille. La véranda arrière est fermée, entièrement persiennée, ce qui s'explique par son orientation à l'Ouest et donc sa forte exposition au soleil de l'après-midi. On aperçoit une cour assez vaste, bordée de plantes caractéristiques des jardins nouméens anciens: taros

24 "M. Lucien Nielly", hommage par le Haut-Commissaire Laurent Péchoux, La France Australe, $\mathrm{n}^{\circ} 20.885$ (23 avril 1959). Voir aussi Annuaire de la Guyane Française et du territoire de l'Inini (Cayenne: Emile Gratien, 1936), p. 74.

25 Témoignages pris auprès de Mmes Carole Sauvan-Nielly et Marie-Josée Nielly, Nouméa, août 2016.

26 Voir Frédéric Angleviel, Maisons nouméennes (Nouméa: Footprint Pacifique, 20I4). 


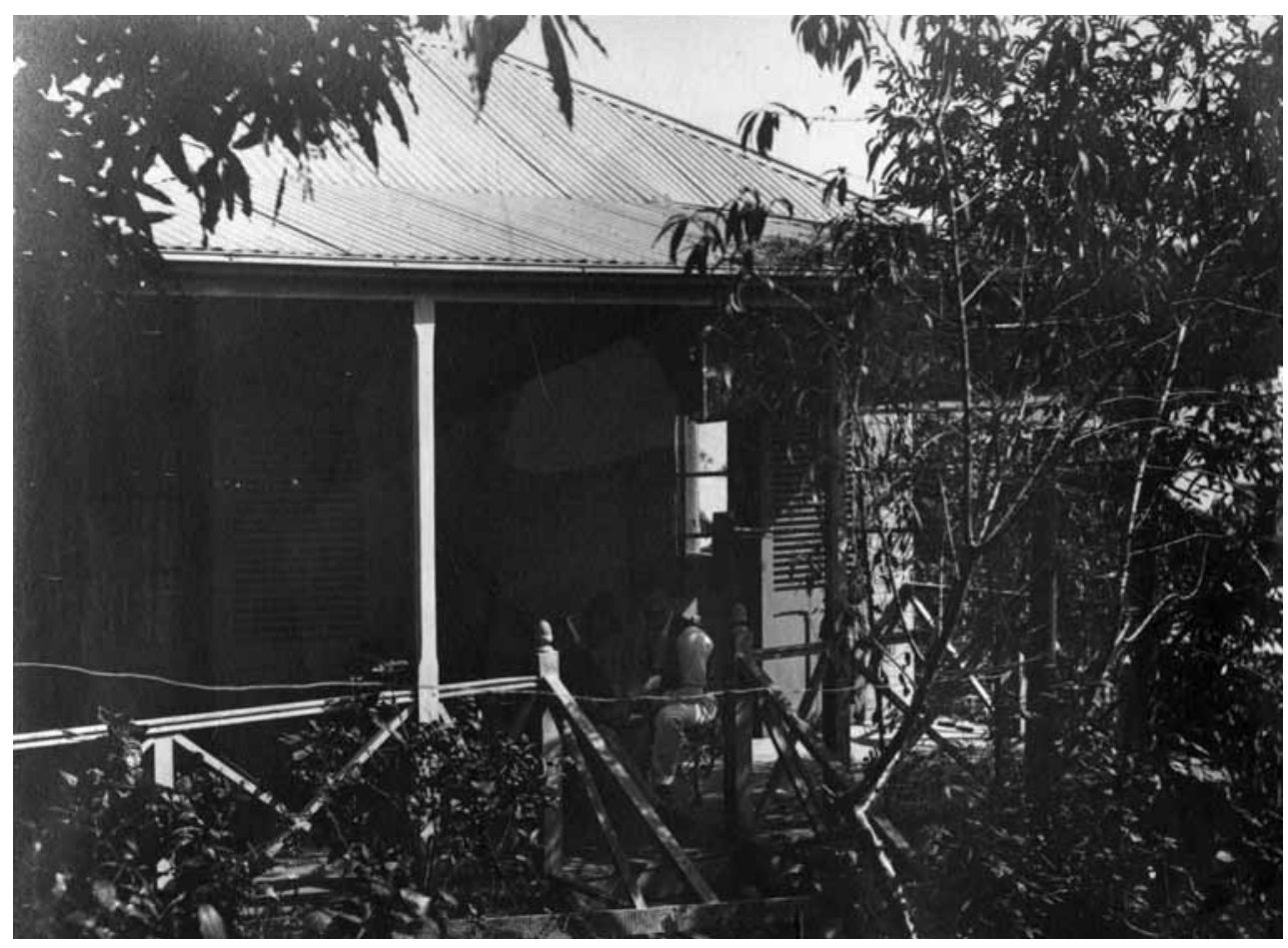

Figure I Mon Home à Nouméa en I902. Photographie albuminée de l'album Nielly, Service des Archives de la Nouvelle-Calédonie, nº I48 Fi5-037.

ornementaux (Alocasia macrorhizza), lauriers-roses (Nerium oleander), niaoulis rouges pleureurs (Callistemon viminalis), ainsi qu'un arbre fruitier aujourd'hui assez rare au premier plan à gauche, le néflier du Japon (Eriobotryca japonica).

En plus d'avoir été un des tous premiers maires de la ville de Nouméa, entre I874 et I879, Arthur Pelletier (I832-r909) était un négociant et homme d'affaires important. ${ }^{27}$ Travaillant notamment de concert avec la compagnie des Messageries Maritimes à laquelle il loue des locaux en $1894,{ }^{28}$ il acquiert progressivement, en périphérie du centre-ville de Nouméa, une série de lots mitoyens sur un même pâté, bordé au Nord par la rue Duquesne, à l'Est par la rue de Sébastopol, au Sud par la rue de Suffren et à l'Ouest par l'avenue de Wagram (actuelle avenue Foch). ${ }^{29}$

27 Patrick O’Reilly, Calédoniens: répertoire bio-bibliographique de la Nouvelle-Calédonie (Paris: Société des Océanistes, I953), p. 205.

28 Registres de formalités hypothécaires du Service de la Publicité Foncière de NouvelleCalédonie, volume 84 , acte 52 .

29 Registre de formalités hypothécaires du Service de la Publicité Foncière de Nouvelle-Calédonie, 


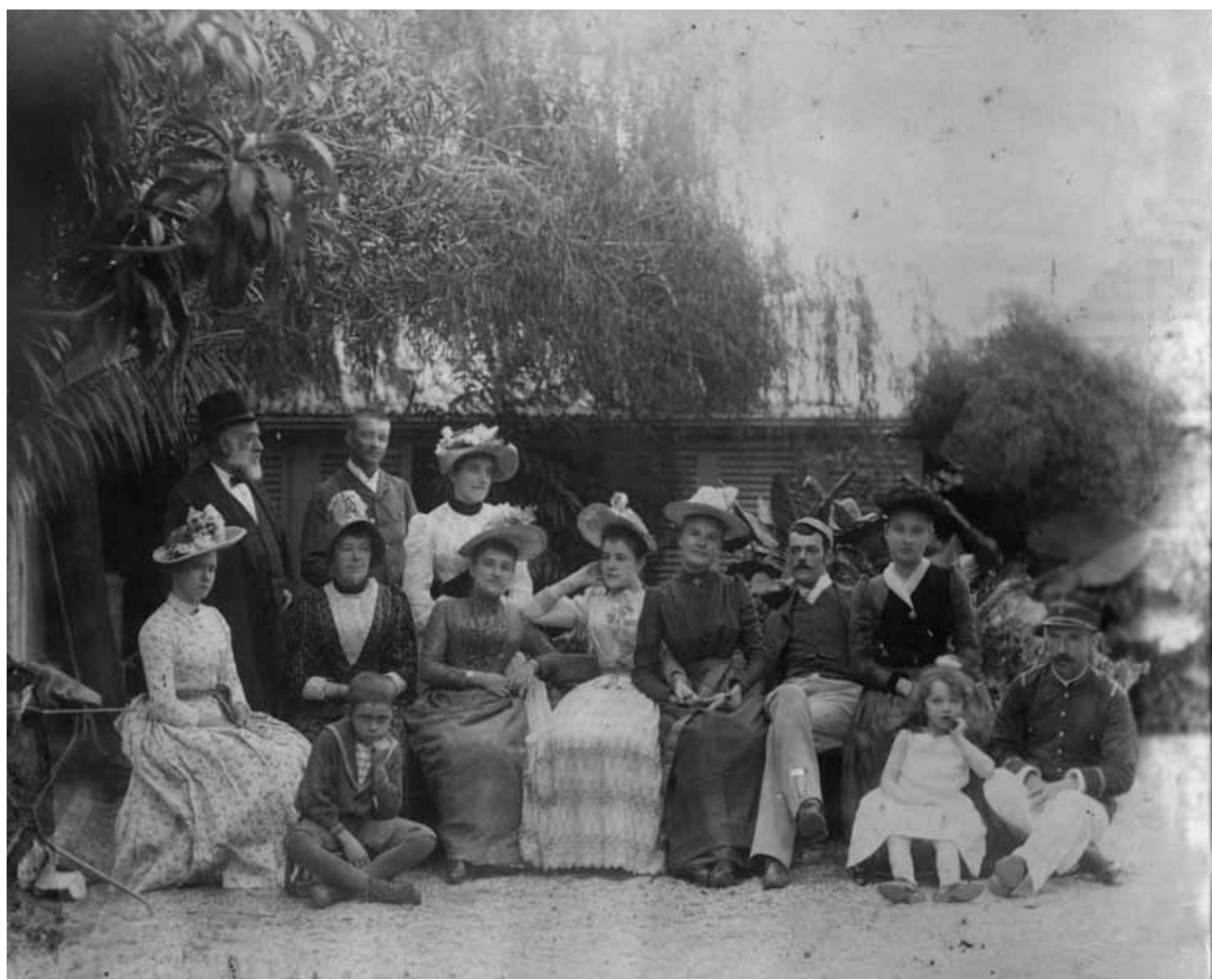

Figure 2 Photographie albuminée de l'album Nielly, Service des Archives de la Nouvelle-Calédonie, no I48 Fi5-059. On reconnaît Arthur Pelletier (de profil à gauche) et son petit-fils Lucien Nielly (à droite, assis entre deux jeunes femmes).

Il lègue ensuite à ses petits-enfants Lucien, Patrice et Madeleine, suite au décès de sa fille et de son beau-fils Charles Nielly, le lot 803 sur le plan de la ville (fig. 3).$^{30}$ Cette localisation, sur les hauteurs du Quartier Latin actuel, est corroborée dans l'album de photographies par plusieurs vues d'une large maison de fonction de la Société le Nickel, située à une cinquantaine de mètres de là et où les frères Nielly figurent à plusieurs reprises (fig. 4). Cette vaste demeure, connue plus tard sous le nom de Chrome Manoir, a été le siège de Radio-Nouméa en Nouvelle-Calédonie. ${ }^{31}$

volume 87 , acte II 4 .

30 Registre de formalités hypothécaires du Service de la Publicité Foncière de NouvelleCalédonie, volume I2, acte 4I, et volume 78 , acte 63 .

3 I Détruite en 1966, on trouve à sa place aujourd'hui les locaux de Radio et Télé NouvelleCalédonie Première. Voir Luc Chevalier, Nouméa rétro 1854-I939: la petite histoire calédonienne, vol. I (Nouméa: Les Éditions du Cagou, I987), p. 74. 


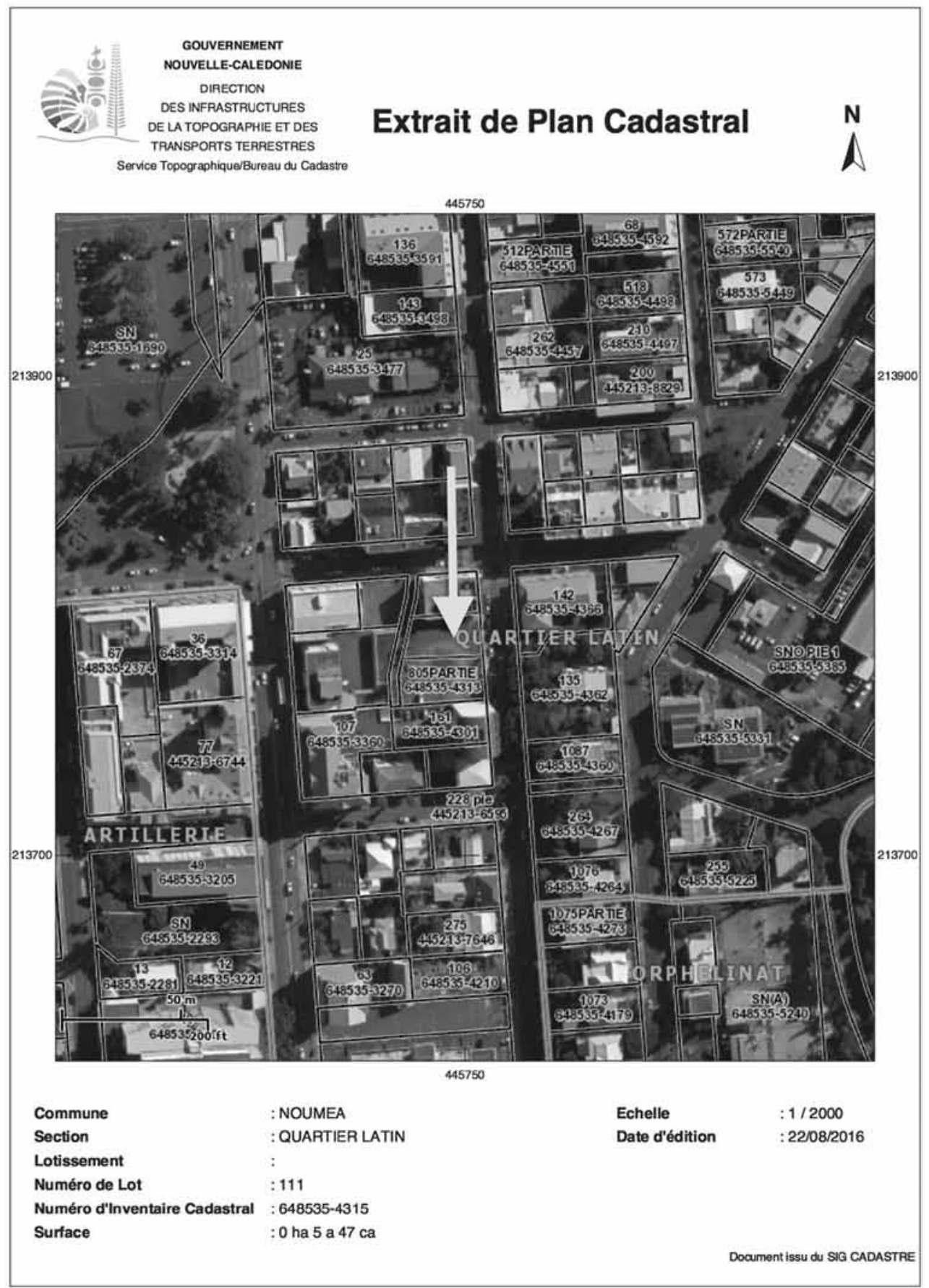

Figure 3 Plan cadastral contemporain du Quartier Latin, Nouméa. La flèche jaune indique le lot 803 , légué par Arthur Pelletier à ses petits-enfants. 


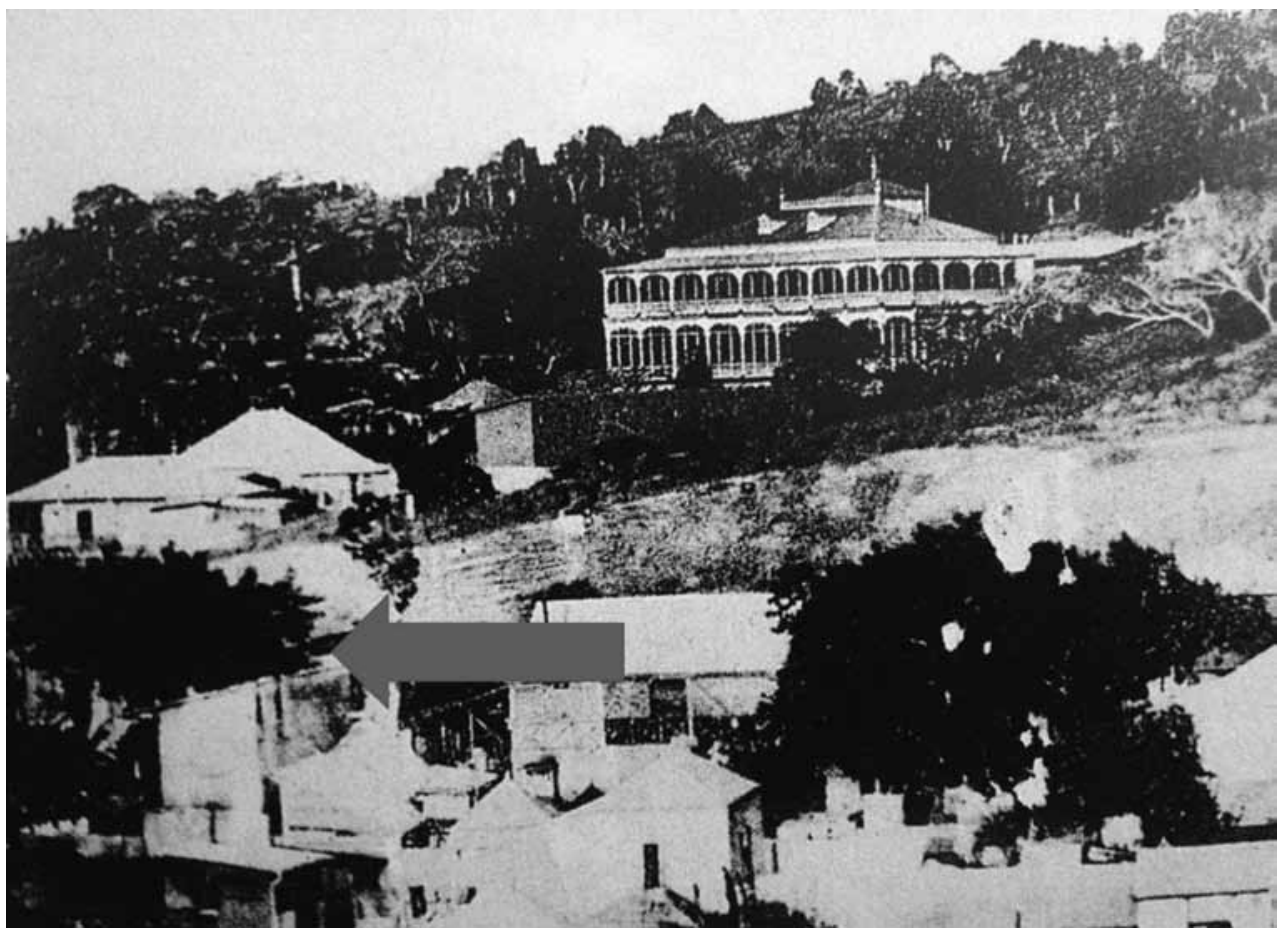

Figure 4 Le Chrome Manoir au début du $20^{\text {ème }}$ siècle (détail d'une carte postale éditée par D. Gubbay, collection particulière,

Nouméa). La flèche indique le lot 803 .

C'est donc en légère périphérie du centre-ville que Victor Segalen s'installe, lors de son second séjour nouméen. On comprend les mentions de bicyclette, ${ }^{32}$ qu'il utilise pour aller à l'hôpital militaire et rendre visite à ses patients, d'autant plus que les frères Nielly, qui ont le même âge, pratiquent ce sport assidûment: Patrice Nielly s'illustre à plusieurs reprises dans des courses cyclistes locales dans les années 1900-1903..$^{33} \mathrm{La}$ maison d'origine n'existe plus aujourd'hui, même si les piliers en brique que surmontait la grille en fonte séparant le terrain de la rue de Sébastopol se dressent toujours, devant un parking vide (fig. 5).

Pourquoi chercher à localiser une maison d'écrivain, en particulier une maison de passage? ${ }^{34}$ Le questionnement archéologique et le questionnement littéraire se rejoignent ici, par l'étude de l'équipement d'un individu qui

32 Segalen, "Deux séjours de Victor Segalen ", p. I87.

33 Jean-Marie Creugnet, Gazette de la Belle Epoque I903 (Mont Dore: Paterna Paternis, 2009).

34 Il s'agit de retrouver ici un lieu, même temporaire, de création. Victor Segalen était d'ailleurs conscient des rapports entre logement et élaboration artistique, en écrivant par exemple: 


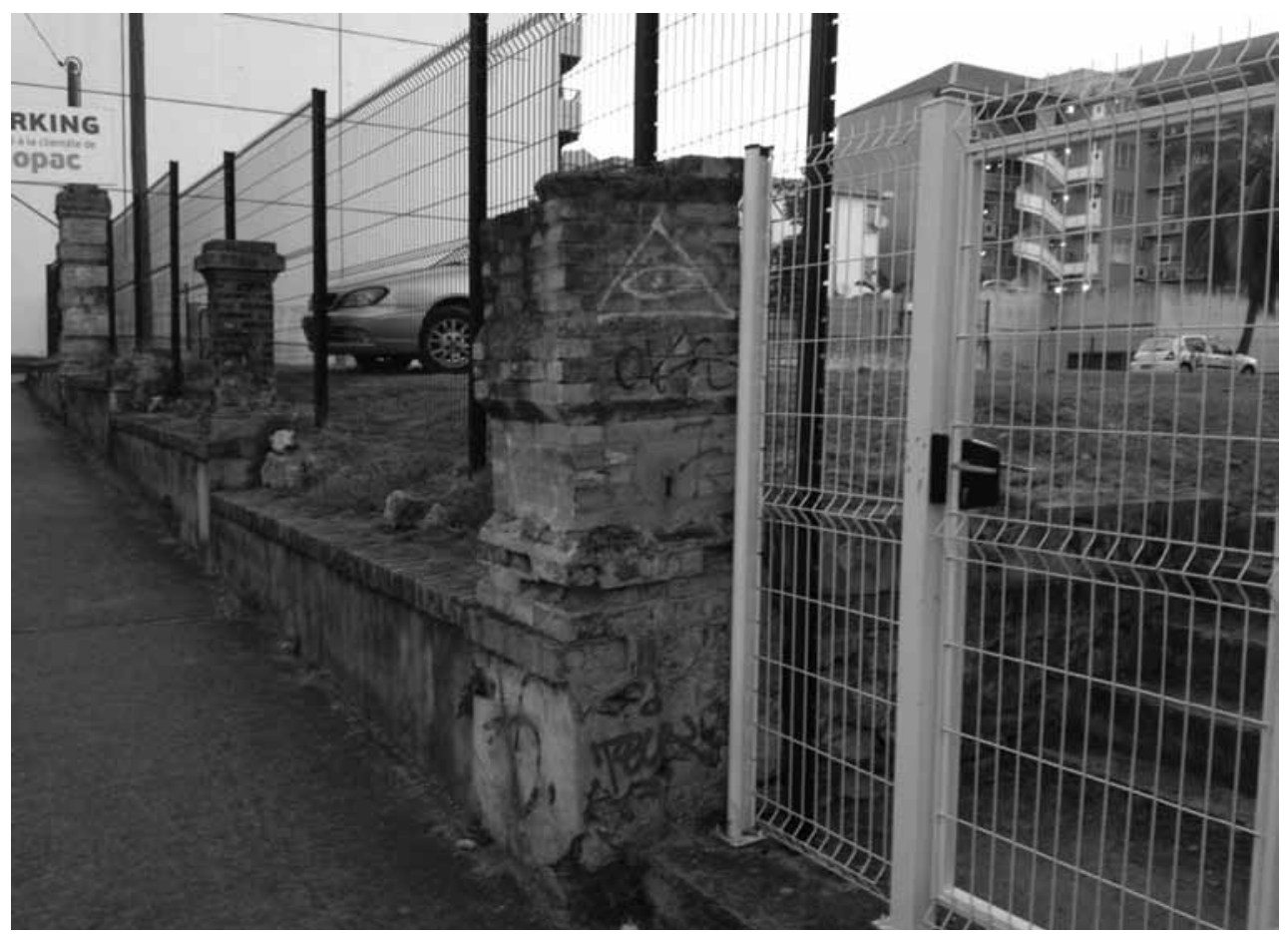

Figure 5 Piliers en briques qui matérialisent l'emplacement de la maison Nielly. On remarque à droite le départ d'un escalier, servitude demandée par Auguste Pelletier au moment de la cession de ce lot à ses petits-enfants.

permet son culte posthume, et par l'étude des conditions d'élaboration d'une œuvre. Archéologie et littérature sont des mots qui entrent aisément en association dans les esprits: le Roman de la Momie de Théophile Gautier ou Salammbô de Gustave Flaubert ont pu être qualifiés de romans archéologiques. ${ }^{35}$ Balzac s'est lui-même qualifié "d'archéologue de la France de son temps ", en ce sens qu'il avait parfaitement compris le rôle de l'archéologue, c'est à dire celui d'un comptable minutieux des artefacts, autrement dit des réalisations techniques. ${ }^{36}$

"De l'artiste à sa demeure, celle-ci n'étant qu'un geste de scène de celui-là ». Victor Segalen, "Gauguin dans son dernier décor ", Le Mercure de France, VI (1904), 679-85 (p. 680).

35 Willhelm Froehner, "Le Roman archéologique en France", Revue contemporaine (3I décembre I862).

36 "L'archéologie comprend l'architecture, la sculpture, la peinture, l'orfèvrerie, la céramique, l'ébénisterie, art tout moderne, les dentelles, les tapisseries, enfin toutes les créations du travail

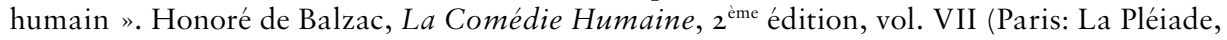
I977), p. 540. Voir également Philippe Bruneau et Pierre-Yves Balut, "Positions ", Revue d'archéologie moderne et d'archéologie générale (RAMAGE), I (I982), 3-33 (pp. 3-II). 
Ici, la démarche archéologique est différente car elle explore la vie de Victor Segalen et n'est pas l'archéologie de son œuvre littéraire océanienne. Ceci serait par exemple le recensement des descriptions archéologiques ou ethnoarchéologiques faites par l'auteur ${ }^{37}$ et partant, l'étude de l'origine de ces renseignements, ou bien encore les modalités techniques de l'écriture comme les choix d'illustrations pris par l'auteur pour Les Immémoriaux par exemple. ${ }^{38}$ Cette étude reste à faire, ${ }^{39}$ et pourra faire l'objet de contributions postérieures, justement quand aura été dépoussiérée au préalable ce qui est ici l'objet de notre étude, à savoir l'archéologie de l'écrivain, ce qui n'est finalement qu'une modalité particulière de l'archéologie de l'individu. ${ }^{40}$ En effet, parce que la personne, en nous, et particulièrement chez l'écrivain, se différencie des autres, il n'y a pas de contradiction fondamentale à faire l'archéologie d'un individu plutôt que celle d'un groupe social (archéologie des Taipi, des paysans d'Indre-et-Loire, de Balzac, etc.). Segalen est ainsi logicien, au sens ou l'entend l'archéologie contemporaine, autrement dit producteur d'un discours technicisé, par ses croquis, ses manuscrits, ses notes raturées (fig. 6).

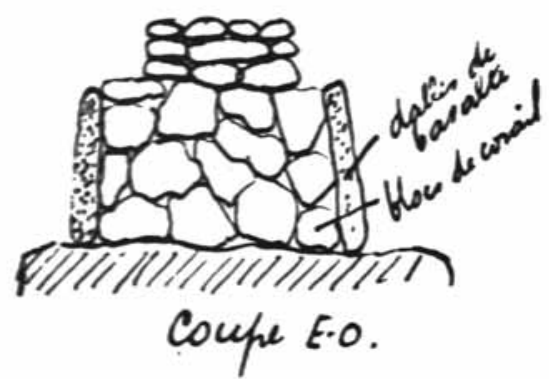

Figure 6 Coupe réalisée au marae de Manunu,

Huahine, le 26 septembre $1903 .{ }^{41}$

37 Et même l'archéologie du propos fictif, ou le fait de retrouver les témoins concrets ayant servi d'inspiration à la création abstraite. Pour une recherche océanienne sur ce thème et la redécouverte d'une maison en lien avec une œuvre littéraire, on consultera Louis Lagarde, 'The Legacy of Planter Jean My: Analysis of Two Private Colonial Homes in Southern Melanesia', Journal of Pacific History, 51.2 (2016), pp. I43-68.

38 Pour une analyse des liens de nature archéologique entre image et légende, voir Philippe Bruneau, "Archéologie et Littérature ", Revue d'archéologie moderne et d'archéologie générale (RAMAGE), II (I993), 55-9I (pp. 67-7I).

39 Un premier travail dans ce sens a été fait grâce à l'inclusion dans l'édition des Immémoriaux de 1956 (Plon, collection Terre Humaine) des notes marginales préparatoires, qui mentionnent les ouvrages de base utilisés par Segalen, et dont une liste figure en bibliographie. Des recoupements avec le travail (ethno-)archéologique de terrain sont à poursuivre.

4I Segalen, Journal des îles, p. 76. 
Il est également technicien, par ses dessins toujours mais aussi par les gestes chirurgicaux qu'il réalise, longuement décrits dans certaines lettres ${ }^{42}$ ou passages du cahier d'observation et de certifications médicales de La Durance. ${ }^{43}$ Au-delà, et comme tout autre, il existe socialement et parce qu'il est personne, il survit à lui-même: l'équipement technique de l'individu, par exemple, les meubles lui ayant appartenu, ses différentes résidences, même passagères, ainsi que sa tombe, intéressent donc l'archéologie. ${ }^{44}$

En termes de recherche littéraire, la maison d'écrivain, voire même ses lieux de séjour provisoire, nous obligent à en reconsidérer l'objet ou, en tout cas, à ré-affronter les problèmes du biographique, a priori tranchés depuis Proust ${ }^{45}$ et l'approche sociocritique. ${ }^{46}$ Ce qui motive en premier lieu la recherche, la visite des lieux de vie et de création des auteurs, relève au départ de l'admiration. Vient ensuite la critique qui permet d'enrichir la connaissance d'une ouvre et de son auteur, le dilemme étant ici de sortir du texte et d'en comprendre les conditions sociales, matérielles et géographiques. De plus, la maison d'un écrivain, ses lieux de séjour, ses repères dans une ville permettent d'augmenter la socialité littéraire d'une œuvre. Associations, cercles de lecteurs ou chercheurs entrent dans un dialogue avec les institutions pour accorder une valeur patrimoniale à un lieu identifié. Celui-ci "développe et diffuse la connaissance d'un écrivain, la connaissance de son œuvre et le fait de multiples manières $»{ }^{47}$ Il s'agit donc ici, d'aborder plus spécifiquement l'expérience de Nouméa par Segalen comme condition d'écriture de son premier roman. Nouméa occupe déjà une place discrète dans le culte qui est aujourd'hui rendu à Victor Segalen: un de ses portraits photographiques les plus utilisés est celui, pris à Nouméa par le photographe et éditeur de cartes postales Louis Talbot (fig. 7).

À l'image du célèbre portrait d'Arthur Rimbaud pris par Etienne Carjat, le cliché de Talbot a figé l'écrivain très jeune (ici à 25 ans). La brièveté de la carrière littéraire de Segalen, mort à 4I ans, a contribué à donner à cette photographie sa puissance évocatrice. Elle matérialise en partie l'échange

42 Segalen, "Deux séjours de Victor Segalen ", pp. I88-89.

43 Segalen, Journal des iles, p. I47.

44 Philippe Bruneau, "Etudes d'archéologie du catholicisme français III ", Revue d'archéologie moderne et d'archéologie générale (RAMAGE), 4 (I986), I27-49 (pp. I37-38).

45 "Le moi de l'écrivain ne se montre que dans ses livres ». Marcel Proust, Contre Sainte-Beuve (Paris: Gallimard, I987 [I954]), p. I33.

46 Claude Duchet, "Pour une sociocritique, ou variation sur un incipit ", Littérature, I.I (I97I), pp. 5-I4.

47 Jean-Pierre Dekiss, "La Maison d'un écrivain, utopie ou enjeu de société », Revue d'histoire littéraire de la France, I09.4 (2009), 783-95 (p. 783). 


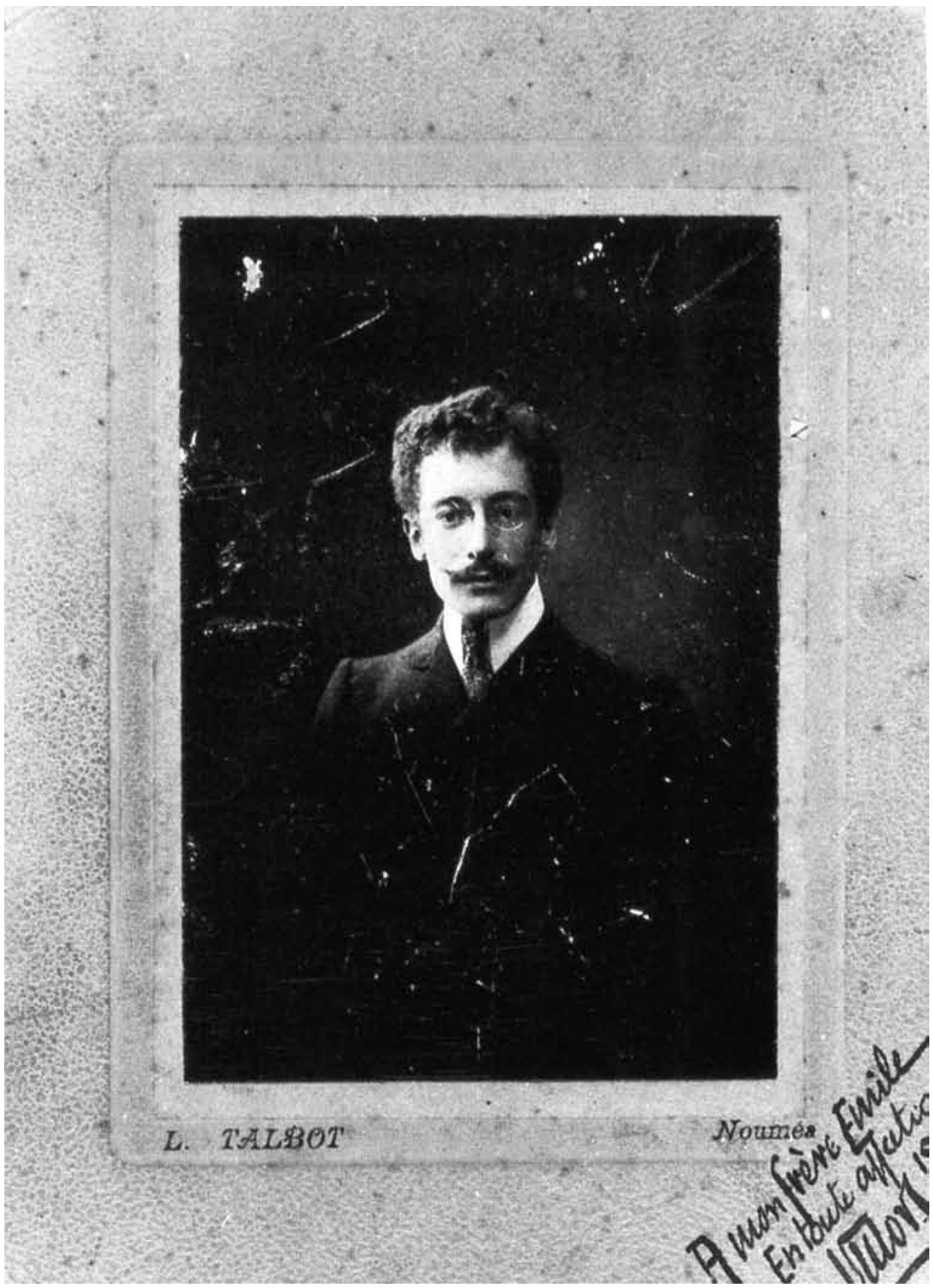

Figure 7 Victor Segalen par Louis Talbot, I903 Ou I904.

Epreuve au gélatino-bromure d'argent sur papier albuminé, contrecollée sur carton. Exemplaire dédicacé à Emile Mignard, destinataire de nombreuses lettres de la Correspondance. 
social entretenu avec lui, c'est à dire le lien perpétué grâce à la place qu'il occupe aujourd'hui parmi les écrivains français passionnés d'exotisme. Ce lien se traduit techniquement, par la dénomination d'institutions (Université Victor Segalen-Bordeaux II, Lycée français international Victor Segalen à Hong Kong), de rues (rue Victor Segalen dans le $20^{\text {ème }}$ arrondissement de Paris), par la fréquentation culturelle de sa tombe (cimetière d'Huelgoat, Finistère) ou de ses lieux de passage.

Les lieux précis de son séjour à Tahiti restent à découvrir mais pourraient être mis en évidence par l'analyse de la correspondance ainsi que, comme nous l'avons fait avec la famille Nielly, par la collecte de traditions orales. Par exemple, les descendants de Ky Dong, déporté politique vietnamien et ami de Paul Gauguin, qui aurait rencontré Segalen lors de ses séjours tahitiens, pourraient renseigner cet aspect de l'étude. ${ }^{48}$ Si Nouméa ne peut pas s'enorgueillir d'avoir frappé l'imaginaire de Victor Segalen, ce dernier lui doit d'y avoir trouvé les conditions de la maturation de son premier chef d'œuvre. À Nouméa, Segalen a travaillé à l'hôpital militaire, ce qui est consigné dans le cahier d'observation et de certifications médicales de La Durance ${ }^{49}$ mais la maison du Quartier Latin où il séjourne et se plonge dans l'écriture est le lieu le plus pertinent pour maintenir le lien patrimonial avec l'auteur. Il serait opportun que la ville de Nouméa valorise ce lieu de passage car la portée littéraire des Immémoriaux tient autant à leur esthétique novatrice qu'au discours politique qu'on a pu y lire, et qui n'est pas sans lien avec les conditions matérielles de leur rédaction.

Le roman polynésien de Victor Segalen pose en effet, depuis sa réédition en 1956 dans la collection Terre Humaine (Plon), ${ }^{50}$ une véritable énigme politique aux chercheurs en littérature: Segalen était-il anticolonial? En effet, dans l'Essai sur l'exotisme, on trouve des notes préparatoires aux Immémoriaux où Segalen annonce vouloir " déblayer ». ${ }^{51}$ Martine AstierLoufti a souligné que "Segalen poursuivait un itinéraire personnel,

O'Reilly, Tahitiens, pp. 580-8I.

Segalen, Journal des îles, pp. I40-53.

Il s'agit du troisième volume de la collection, après celui du fondateur Jean Malaurie, Les Derniers rois de Thulé (1955), et Tristes Tropiques (1955) de Claude Lévi-Strauss.

I Les dernières lignes de "Gauguin dans son dernier décor " (op. cit.) constituent déjà un réquisitoire anticolonial: "Tout vit, tout surgit, dans la tiédeur parfumée des étés à peine nuancés de sécheresse, tout: hormis la race des hommes. Car ils agonisent, ils meurent, les pâles Marquisiens élancés. Sans regrets, sans plaintes ni récris, ils s'acheminent vers l'épuisement prochain. Et là encore, à quoi serviraient de pompeux diagnostics? L'opium les a émaciés, les terribles jus fermentés les ont corrodés d'ivresses neuves; la phtisie creuse leurs poitrines, la syphilis les tare d'infécondité. Mais qu'est-ce que tout cela sinon les modes divers de cet autre fléau: le contact des 'civilisés'. Dans vingt ans ils auront cessé d'être 'sauvages'. Ils auront, en même temps, à jamais, cessé d'être »(p. 684). 
spirituel et esthétique qui se plaçait en dehors des incidences politiques, il n'était absolument pas question qu'il développât sur le plan de l'action anticolonialiste, les principes de sa philosophie de l'exotisme $" .{ }^{52}$ Christian Doumet a montré que Les Immémoriaux relevaient de la "tentation d'un langage-autre ${ }^{53}$ dont les prolongements sont presque immédiatement politiques dans la mesure où le roman s'oppose à son époque. Inspiré par Rimbaud, il se livre en effet à une réinvention poétique de soi en adoptant une conscience maorie et fait entendre à son lecteur une voix indigène contemptrice du trauma colonial de l'évangélisation. C'est la marque d'une réflexion esthétique singulière et l'Essai sur l'exotisme (rédigé entre 1903 et I905), publié à titre posthume en I955, montre que l'écriture du roman fut également motivée par une volonté d'approfondir cette notion. Le retour, à partir de la correspondance sur la présence de Segalen à Nouméa, incite à interpréter les stratégies sociales qui ont présidé à l'écriture d'un texte qui, observe encore Astier-Loutfi, " fut une réplique proprement littéraire à l'impérialisme ». ${ }^{54}$ Henri Lavondès note ainsi que "Les Immémoriaux sont un cri, un cri prématuré, faute d'oreilles capables de l'entendre, un cri poussé cinquante ans trop tôt dans le désert d'un siècle que passionnait l'aventure coloniale, et d'une civilisation beaucoup trop sûre encore de ses propres valeurs pour être capable de reconnaître celles des autres $»{ }^{55}$ De même, Jean-François Mattéi juge qu'" il suffit de lire Les Immémoriaux de Victor Segalen pour épouser la mauvaise conscience de l'homme occidental ». ${ }^{56} \mathrm{La}$ singularité des Immémoriaux vient en effet précisément de la rupture qu'ils opèrent avec le roman colonial alors en vogue où l'exotisme était prétexte à la célébration de l'empire colonial français.

Toutefois, revenant sur l'impact de la publication de la correspondance de l'auteur, le romancier Simon Leys tempère cette interprétation: "malgré tous les dons d'intelligence et de cœur, Segalen était aussi, qu'il le veuille ou non, un enfant stupide du XIX ${ }^{\mathrm{e}}$ siècle $» .{ }^{57}$ Les commentaires sur sa vie à Tahiti et à Nouméa révèlent en effet un personnage volontiers méprisant, partageant le racialisme de son temps. En nous penchant sur le lieu du

52 Martine Astier-Loufti, Littérature et colonialisme. L'expansion coloniale vue dans la littérature romanesque française I87I-I9I4 (Paris: Mouton, I97I), p. I35.

53 Christian Doumet, "Ecriture de l'exotisme: Les Immémoriaux de Victor Segalen", Littérature, 5I (I983), 9I-IO3 (p. 97).

54 Astier-Loufti, p. I32.

55 Henri Lavondès, "À la recherche de la mémoire perdue, Les Immémoriaux, Le Maître du jouir et les Polynésiens d'aujourd'hui ", dans Victor Segalen / recueil de textes, dir. par Marie Dollé et Christian Doumet (Paris: L'Herne, 1998), 269-78 (p. 269).

56 Jean-François Mattéi, Le Procès de l'Europe: grandeur et misère de la culture européenne (Paris: Presses Universitaires de France, 20II), p. II3.

57 Simon Leys, Studio de l'inutilité (Paris: Flammarion, 20I2), p. I27. 
séjour nouméen de Segalen, on peut mettre en avant la sociabilité coloniale vécue par l'auteur. Ainsi, le toponyme du Quartier Latin où il a séjourné et qui abritait les premières maisons privées de ce qui était alors une colonie pénitentiaire, trahit la volonté coloniale de reproduire une vie parisienne. Logé avec Lucien Nielly, fonctionnaire, chez le grand-père de ce dernier, premier maire de Nouméa, Segalen s'installe ici dans une situation ambivalente: le mépris relatif qu'il éprouve pour ses hôtes entre en contradiction apparente avec l'acceptation de leur offre d'hospitalité. ${ }^{58}$ À ce titre, en dépit des accents anticoloniaux qu'on a pu lui trouver, il faut souligner que la composition des Immémoriaux a été rendue possible par le confort d'une situation de fonctionnaire colonial: celle d'un médecin de marine affecté en Polynésie, qui pouvait dans les deux archipels, trouver dans son réseau de connaissances et les sociétés coloniales établies, l'équipement nécessaire à son travail littéraire.

\section{Conclusion}

L'aventure océanienne de Victor Segalen n'aurait probablement pas été semblable sans La Mariposa ni La Durance, les navires qui le mènent tour à tour de San Francisco à Tahiti, puis de Tahiti à Toulon. S'il a pu s'imprégner de l'Océanie et de ses particularismes, c'est parce que les missions se sont multipliées dans le cadre d'une Océanie francophone déjà densément interconnectée grâce aux efforts consentis par la puissance coloniale pour le maintien de son autorité. De cette aventure émergent plusieurs œuvres, parmi lesquelles Les Immémoriaux, dont la séquence génératrice peut être décomposée en deux phases: l'une européenne et tardive, entre la fin de I906 et I907, et l'autre océanienne en I903-I904 où le rôle de Nouméa doit dorénavant être reconsidéré. Il est ainsi frappant de constater que le monde des fonctionnaires de l'administration coloniale a été le creuset d'un des romans les plus audacieux sur la colonisation du Pacifique. On perçoit même comment cet épisode de création calédonienne, qui correspond à un éloignement dans une sorte de France australe, est finalement voisin de la phase métropolitaine de rédaction. Il a en quelque sorte fallu, pour Segalen, quitter la Polynésie pour mieux la décrire, prolonger son séjour

58 S'il a pu qualifier Lucien Nielly de «naïf " et de "puéril» (Segalen, Correspondances, vol. I, p. 578), il le décrit aussi comme "l'ami Nielly, toujours très dévoué », qui lui est "précieux" (Segalen, "Deux séjours de Victor Segalen ", p. I86). Il ajoute qu'en ville il a « de bons camarades et quelques bonnes relations " (Segalen, "Deux séjours de Victor Segalen ", p. I87). 
esthétique et intellectuel par le deuil de ne plus y vivre concrètement, seule condition à la maturation de son œuvre..$^{59}$

Le lieu océanien d'écriture des Immémoriaux nous serait resté abstrait sans la publication de la correspondance à partir de laquelle nous avons pu reconstituer un espace propice à la création littéraire: la maison de Lucien Nielly. L'effacement de Nouméa dans le Cycle polynésien est peut-être le signe de l'aversion de Segalen ou, plus simplement le fait d'un projet esthétique - un récit dans le Tahiti du début du ${ }^{2} 9^{\text {ème }}$ siècle - qui laissait peu de place à la petite bourgade coloniale. Par contraste, il décrira dans Equipée ${ }^{60}$ la « chambre aux porcelaines » qu'il occupait pendant ses missions chinoises en I9I4 afin d'" en finir avec [... l'emprise du bon gros Réel ", ${ }^{11}$ l'évocation de ses conditions matérielles aidant ici à se plonger dans l'écriture. Au Quartier Latin, l'emplacement de la maison Nielly et ses éléments encore visibles constituent, grâce à la renommée contemporaine de Segalen, un ensemble important tant du point de vue des études littéraires que de l'archéologie de la personne. Cependant, son accession au statut de relique ne peut se faire sans sa reconnaissance publique: en effet toute relique n'est jamais qu'un témoignage anecdotique, sélectionné arbitrairement par un groupe ou une société. ${ }^{62}$ À Tahiti et à Samoa, les habitations de James Norman Hall et de Robert Louis Stevenson sont aujourd'hui restaurées et visitées. En Nouvelle-Calédonie, aucune plaque n'orne encore les lieux où se trouvaient les maisons natales de Francis $\mathrm{Carco}^{63}$ ou de Jean Mariotti, ni ceux où ont pu être périodiquement stationnés, pendant la Seconde Guerre Mondiale, James A. Michener ou Edgar Rice Burroughs. ${ }^{64}$ C'est l'instauration, par

59 À la suite de cet article, un important travail de recherche génétique reste à mener, notamment à travers dans le fonds Segalen de la Bibliothèque Nationale de France, en particulier grâce aux dossier Mythes polynésiens, qui comporte de nombreux éléments préparatoires aux Immémoriaux. Ici, le propos était de poser les conditions de l'écriture de l'œuvre: d'une part en l'ancrant physiquement dans le Pacifique à travers la recherche d'un lieu important de création, et d'autre part en démontrant qu'elle est rendue possible par l'existence de la francosphère Pacifique, les allers-retours de La Durance permettant l'éloignement puis le rapprochement incessant de l'auteur vis-à-vis de son objet d'étude.

60 Publié à titre posthume en I929.

6I Dans ce passage, Ségalen évoquait également "les mauvais doutes " inhérents tant aux préparatifs de l'expédition archéologique qu'à la création littéraire. Victor Segalen, Equipée (Paris: Gallimard, I983 [I929]), p. I8.

62 Philippe Bruneau et Pierre-Yves Balut, Artistique et Archéologie (Paris: Presses Universitaires de Paris-Sorbonne, I997), p. I30.

63 Paul Griscelli se plaignait déjà du peu de reconnaissance locale attribué à Francis Carco, pourtant natif de Nouméa et membre de l'Académie Goncourt (Paul Griscelli, 1988). Une stèle a pendant quelques temps marqué l'emplacement de sa maison: elle a été rapidement ôtée et est aujourd'hui visible dans le jardin de la Maison du Livre de Nouméa.

64 Si la valorisation d'une maison d'écrivain peut se comprendre par l'attrait touristique (et les avantages financiers afférents) que celle-ci représente, elle participe avant tout du maintien ou de la création d'un lien patrimonial entre la société contemporaine et l'auteur dont celle-ci, 
choix politique, d'un lien patrimonial avec ce lieu qui peut, malgré la destruction passée du bien, permettre à la Nouvelle-Calédonie de renouer des liens avec le personnage passionnant que fut Segalen. Il est peut-être temps en effet que ce pays valorise les traces du passage des écrivains, pour s'approprier ces derniers.

ainsi, se revendique. Tout patrimoine n'est au final que «ce qu'une société accepte ou refuse d'une autre pour se construire elle-même ». Pierre-Yves Balut, "Du patrimoine ", Revue d'archéologie moderne et d'archéologie générale (RAMAGE), 2 (1983), 207-37 (p. 209). 